\title{
QUALIDADE DE CHAPAS DE PARTÍCULAS DE MADEIRA AGLOMERADA FABRICADAS COM RESÍDUOS DE UMA INDÚSTRIA DE CELULOSE
}

\section{QUALITY OF WOOD PARTICLEBOARD MANUFACTURED WITH RESIDUES FROM A PULP FACTORY}

\author{
Cristiane Pedrazzi $^{1}$ Clovis Roberto Haselein ${ }^{2}$ Elio José Santini ${ }^{3}$ Paulo Renato Schneider ${ }^{4}$ \\ RESUMO
}

Este trabalho teve como objetivo avaliar a qualidade de chapas aglomeradas fabricadas com resíduos da madeira de Eucalyptus saligna, resultantes da picagem das toras para a confecção de cavacos utilizados na produção de celulose. As chapas foram produzidas com dois tipos de resíduos, palitos e serragem, sendo utilizados puros. $\mathrm{O}$ adesivo usado foi à base de uréia-formaldeído em proporções de 4; 8 e 12\%, com base na massa seca das partículas, e as densidades nominais das chapas foram de 0,$55 ; 0,65$ e $0,75 \mathrm{~g} / \mathrm{cm}^{3}$. Foram avaliadas propriedades de flexão estática, ligação interna, arrancamento de parafusos, absorção d'água e inchamento em espessura. Os valores obtidos das propriedades de flexão estática, ligação interna e arrancamento de parafusos aumentaram com a densidade da chapa, assim como com o teor de adesivo, independentemente do tipo de resíduo. No caso da absorção d'água, os valores aumentaram com a redução da densidade, tanto para chapas produzidas com partículas tipo palitos como para serragem. Para inchamento em espessura, os valores aumentaram com a diminuição do teor de adesivo, sem considerar o do tipo de resíduo utilizado. Os resultados permitem concluir que, usando-se tanto partículas tipo palitos quanto serragem com maiores percentagens de adesivo e maiores densidades, podem ser produzidas chapas com qualidade satisfatória.

Palavras-chave: chapas aglomeradas; Eucalytus saligna; uréia-formaldeído; resíduos de madeira.

\begin{abstract}
The objective of this work was to investigate the quality of particleboards made with Eucalyptus saligna wood residues, resulting from the chiping of logs for pulp production. The boards were producted with two types of residues, sticks and sawdust, used pure. The adhesive used was urea-formaldehyde resin in proportions of 4,8 and $12 \%$ (based on ovendry weight of wood particles) and the nominal densities were $0.55,0.65$ and $0.75 \mathrm{~g} / \mathrm{cm}^{3}$. Bending properties, internal bond, screw withdrawal, water absorption and thickness swelling ware evaluated. The results indicated that the bending properties, internal bond and screw withdrawal were slightly higher with the sawdust type residue than boards made with sticktype residue. For water absorption, the values increased with the reduction of the density, for both type of particles. Thickness swelling increased with the reduction of resin proportion, independent of the type of residue used. Results showed that using both particles type with larger resin proportion and larger densities, boards with acceptable quality can be made.
\end{abstract}

Keywords: particleboard; Eucalyptus saligna; urea formaldehyde; residues of wood. INTRODUÇÃO

Em conseqüência da grande diversidade e quantidade de florestas naturais tropicais no Brasil, o surgimento de indústrias madeireiras é cada vez mais intenso. Porém, pela inadequada exploração e utilização não-racional dessas florestas, associada ao aumento da demanda de produtos madeireiros, torna-se interessante o uso de novas formas de aproveitamento de resíduos de madeira, oriundas do sistema industrial de fabricação de celulose e papel e de serrarias.

Com isso, a indústria madeireira deve aperfeiçoar-se para manter sua competitividade no mercado.

1. Engenheira Florestal, Professora Substituta do Departamento de Química, Centro de Ciências Naturais e Exatas, Universidade Federal de Santa Maria, CEP 97105-900, Santa Maria (RS). cpedrazzi@terra.com.br

2. Engenheiro Florestal, PhD., Professor Adjunto do Departamento de Ciências Florestais, Centro de Ciências Rurais, Universidade Federal de Santa Maria, CEP 97105-900, Santa Maria (RS). haseleic@ccr.ufsm.br

3. Engenheiro Florestal, Dr., Professor Adjunto do Departamento de Ciências Florestais, Centro de Ciências Rurais, Universidade Federal de Santa Maria, CEP 97105-900, Santa Maria (RS). santinie@ccr.ufsm.br

4. Engenheiro Florestal, Dr., Professor Titular do Departamento de Ciências Florestais, Centro de Ciências Rurais, Universidade Federal de Santa Maria, CEP 97105-900, Santa Maria (RS).paulors@ccr.ufsm.br

Recebido para publicação em 26/04/2005 e aceito em 4/05/2006. 
Assim, a redução de perdas, a automação do processo com utilização de novos equipamentos e o desenvolvimento de novas tecnologias e produtos passaram a ser questão fundamental à sobrevivência a médio e longo prazo. Por isso, atualmente as indústrias madeireiras estão adotando novas tecnologias para obter um melhor e maior aproveitamento da matéria-prima.

A tendência de utilização de resíduos fibrosos é crescente, pois, além de servir como matéria-prima para as indústrias, também possibilita uma produção ecologicamente eficiente e contribuindo na melhoria e na qualidade do produto final.

Segundo Iwakiri et al.(1999a), as grandes áreas de reflorestamentos de eucalipto foram implantadas com a finalidade de suprir as demandas de indústrias siderúrgicas e para, posteriormente, serem utilizadas na produção de cavacos destinados às indústrias de celulose e papel e de painéis de madeira.

Para Dacosta (2004), a matéria-prima pode resultar de madeira extraída dos reflorestamentos, bem como do aproveitamento de resíduos industriais florestais ou de agricultura com a finalidade direta de abastecer as indústrias de chapas aglomeradas.

Espécies de Eucalyptus spp e Pinus spp apresentam grande potencial para suprir essa demanda em razão das boas características da sua madeira, por serem espécies de rápido crescimento e também por causa da disponibilidade de grandes áreas plantadas no Brasil (Iwakiri et al., 2000).

O sucesso do uso do eucalipto para a obtenção de chapas de fibras no Brasil é demonstrado pelo simples fato de que praticamente toda a nossa produção de chapas está baseada na utilização de madeira obtida de plantações desse gênero. As espécies mais utilizadas para essa produção têm sido o Eucalyptus grandis e o Eucalyptus saligna (Brito et al.,1998).

As principais fontes de resíduos são as serrarias e os setores de picagem da madeira em fábricas de celulose e papel. Em ambos os casos, os resíduos fibrosos são separados e acumulados. A serragem de serrarias é heterogênea quanto à composição, pois serrarias trabalham geralmente com tipos diversos de madeira. Já a serragem da indústria de celulose é separada na classificação dos cavacos, sendo conhecida como "finos" (Foelkel et al., 1979).

Quanto aos principais adesivos empregados na fabricação de painéis à base de madeira, destacam-se os adesivos sintéticos, como o fenol-formaldeído, o resorcinol-formaldeído, a uréia-formaldeído e a melamina-formaldeído (Koch et al.,1987).

A resina de uréia é a mais empregada por razões econômicas e tecnológicas. A manufatura de chapas de boa qualidade com o menor consumo de adesivo é o objetivo primordial dos fabricantes, em razão sobretudo do alto custo representado por ela (Iwakiri et al., 1995).

Uma das variáveis de processo na produção de painéis de madeira aglomerada é a espécie da madeira e a conseqüente relação entre a densidade do painel e a densidade da madeira utilizada, denominada de razão de compactação (Kelly, 1977; Moslemi, 1974; Maloney, 1993; Tsoumis, 1991).

A utilização de madeiras de baixa densidade resulta em alta razão de compactação das chapas e maior área de contato entre as partículas, resultando em melhores propriedades de flexão estática e ligação interna (Kelly, 1977).

Estudos realizados recentemente por Calegari et al. (2004) revelaram que o teor de adesivo afetou significativamente tanto a resistência à flexão, bem como a ligação interna e o inchamento em espessura das chapas. Ocorreu uma diminuição da qualidade dos painéis pela redução do teor de adesivo.

Pizzi (1994) também verificou que, com o aumento do teor de resina, independentemente de seu tipo, ocorre um aumento dos valores de propriedades mecânicas e da estabilidade dimensional dos painéis.

O presente estudo foi realizado com o objetivo de analisar a viabilidade do uso de resíduos fibrosos de Eucalyptus saligna, oriundos de uma indústria de celulose na fabricação de chapas de madeira aglomerada. 


\section{MATERIAL E MÉTODOS}

\section{Fabricação das chapas}

A matéria-prima utilizada na confecção das chapas de aglomerado foi coletada do abastecimento da indústria de celulose e papel Klabin Riocell, localizada em Guaíba, RS. Era constituída de resíduos dos cavacos usados na produção de celulose de madeira de Eucalyptus saligna.

O resíduo era constituído por pedaços de cavacos, palitos, serragem e pó. Posteriormente, esse resíduo foi peneirado para a separação e classificação das partículas em palitos e serragem e para a remoção do pó, que foi descartado. A peneira utilizada para a separação do palito da serragem tinha malha com aberturas de 2,51 x 2,51 mm e, para a separação da serragem do pó, de 1,42 x 1,42 mm. A separação dos pedaços de cavacos dos palitos foi realizada manualmente.

Os palitos obtidos tinham dimensões médias de $18,7 \times 3,15 \times 1,04 \mathrm{~mm}$, e a serragem, valores médios de 9,25 x 1,47 x $0,62 \mathrm{~mm}$.

Em seguida, os palitos e a serragem foram para a estufa a $60^{\circ} \mathrm{C}$, com circulação de ar forçada para secagem, até atingir um teor de umidade de aproximadamente $3 \%$.

$\mathrm{O}$ adesivo empregado foi a base de uréia formaldeído. Para determinar o teor de sólidos da resina uréia formaldeído e da parafina, aplicou-se o procedimento da norma ASTM D 1490-96 (1999)

Foram confeccionadas 54 chapas aglomeradas, com dimensões de 50 x 50 x 0,95 cm.

As variáveis de estudo para cada tratamento foram o tipo de partícula utilizada(palito e serragem) a densidade da chapa $\left(0,55,0,65\right.$ e $\left.0,75 \mathrm{~g} / \mathrm{cm}^{3}\right)$ e o teor de adesivo $(4,8$ e $12 \%)$. Também foram inseridos outros fatores no processo produtivo que foram fixos para os tratamentos.

A prensagem a quente foi realizada em prensa de laboratório, hidráulica com pressão específica aplicada de $30 \mathrm{kgf} / \mathrm{cm}^{2}$ e temperatura dos pratos ajustada para $180^{\circ} \mathrm{C}$. O tempo de fechamento da prensa foi de 40 segundos, e o tempo total de prensagem foi de 10 minutos.

Depois da prensagem, as chapas foram identificadas, esquadrejadas e conduzidas à câmara climática para serem condicionadas à temperatura de $20^{\circ} \mathrm{C}$ e umidade relativa de $65 \%$.

\section{Testes mecânicos}

Foram avaliadas, pela norma norte-americana D-1037-93 (ASTM, 1995), as seguintes propriedades mecânicas das chapas: flexão estática como o módulo de ruptura (MOR) e módulo de elasticidade (MOE), arrancamento de parafusos (AP) e ligação interna (LI) e de propriedades físicas: absorção d'água e inchamento em espessura.

Para realizar o teste de arrancamento de parafusos (AP), fez-se uso de corpos de prova de 15 x 7,5 cm, aproveitando as extremidades dos corpos de prova utilizados no teste de flexão.

Para realizar o teste de ligação interna (LI), foram utilizados corpos de prova de $5 \times 5 \mathrm{~cm}$, com as faces coladas em blocos de aço. Foram medidas as dimensões da área de esforço e, no momento da ruptura, registrou-se a carga máxima.

Nos testes de estabilidade dimensional (absorção d'água e inchamento em espessura), foram utilizados corpos de prova de $15 \times 15 \mathrm{~cm}$ que formam submersos em água sob temperatura de $20^{\circ} \mathrm{C}$. Cada corpo de prova foi pesado, e a espessura foi medida antes de serem mergulhados na água e após 24 horas de submersão.

\section{Modelo experimental e análise dos resultados}

As combinações empregadas para a confecção das chapas de partícula (Tabela 1) foram dois tipos de partículas definidas como palitos e serragem, três níveis de adesivo e três densidades. Para cada tratamento, foram realizados três repetições.

Os resultados obtidos foram analisados pelo teste de correlação de médias e análise de regressão, relacionando-se os resultados dos testes com as variáveis do estudo.

A seleção dos melhores modelos foi realizada escolhendo os menores valores de P, Syx (erro-padrão 
da estimativa), e os maiores valores de $\mathrm{F}, \mathrm{R}^{2}$ ajustado (coeficiente de determinação), além da análise do gráfico do resíduo.

TABELA 1: Tratamentos que foram utilizados na confecção das chapas.

TABLE 1: Treatments used for the manufacturing of particleaboards.

\begin{tabular}{c|c|c|c}
\hline Tratamentos & Tipo de partícula & Teor de adesivo $(\%)$ & Densidade $\left(\mathrm{g} / \mathrm{cm}^{3}\right)$ \\
\hline 1 & Palito & 4 & 0,55 \\
2 & Palito & 8 & 0,55 \\
3 & Palito & 12 & 0,55 \\
4 & Palito & 4 & 0,65 \\
5 & Palito & 8 & 0,65 \\
6 & Palito & 12 & 0,65 \\
7 & Palito & 4 & 0,75 \\
8 & Palito & 8 & 0,75 \\
9 & Palito & 12 & 0,75 \\
10 & Serragem & 4 & 0,55 \\
11 & Serragem & 8 & 0,55 \\
12 & Serragem & 12 & 0,55 \\
13 & Serragem & 4 & 0,65 \\
14 & Serragem & 8 & 0,65 \\
15 & Serragem & 12 & 0,65 \\
16 & Serragem & 4 & 0,75 \\
17 & Serragem & 8 & 0,75 \\
18 & Serragem & 12 & 0,75 \\
\hline
\end{tabular}

\section{RESULTADOS E DISCUSSÃO}

\section{Propriedades mecânicas}

\section{Resistência à flexão estática (MOR e MOE), ligação interna (LI) e arrancamento de parafusos (AP)}

A norma de comercialização ANSI A 208.1 das chapas de partículas de baixa e média densidade (menor que 0,59 e entre 0,59 e $0,8 \mathrm{~g} / \mathrm{cm}^{3}$ respectivamente) admite como valores mínimos requeridos 56 e $112 \mathrm{kgf} / \mathrm{cm}^{2}$ de MOR e 10561 e $17602 \mathrm{kgf} / \mathrm{cm}^{2}$ para MOE, 1,40 e 4,22 $\mathrm{kgf} / \mathrm{cm}^{2}$ para LI e, 61 e $102 \mathrm{kgf}$ para AP.

Os valores médios dos ensaios em flexão (MOR e MOE), LI, AP, absorção d'água e inchamento em espessura, obtidos para as chapas estudadas, são apresentados na Tabela 2, em função do tipo de partícula utilizada palito e serragem), teor de adesivo e densidade. Pode-se observar que os valores de MOR oscilaram entre 38,08 e $95,84 \mathrm{kgf} / \mathrm{cm}^{2}$ e MOE entre 5048 e $16206 \mathrm{kgf} / \mathrm{cm}^{2}$ para chapas produzidas com partícula tipo palito. Para chapas produzidas com partícula tipo serragem os valores de MOR, variaram entre 42,76 e $100,86 \mathrm{kgf} / \mathrm{cm}^{2}$ e para MOE, entre 7351 e $18574 \mathrm{kgf} / \mathrm{cm}^{2}$. Já para a LI e AP, os valores oscilaram entre 0,36 e $3,04 \mathrm{kgf} / \mathrm{cm}^{2}$, e entre 34 e $80 \mathrm{kgf}$ respectivamente, tanto para chapas fabricadas com partícula tipo palito quanto para serragem.

Os valores obtidos para os ensaios de MOR e MOE, na maioria dos tratamentos, superaram o valor mínimo requerido. Também foi verificado que os melhores valores foram os obtidos com as chapas com maior teor de adesivo e maior densidade.

Da mesma forma, Dacosta (2004), Hillig (2000) e Haselein (1989) observaram que, com o aumento do teor de adesivo e da densidade das chapas, os valores de MOR e MOE aumentam.

Também se constatou neste estudo que, os maiores valores de MOR e MOE foram obtidos com as chapas fabricadas com partículas tipo serragem. Isso foi constatado pois, comparadas às partículas tipo palito, as partículas serragem são mais finas e menos espessas, e dessa maneira, mais flexíveis, o que favorece a colagem e a estrutura final da chapa. 
TABELA 2: Valores médios de MOR, MOE, LI, AP, Ab 24 e IE 24 encontrados para os diferentes tratamentos.

TABLE 2: Average values of MOR, MOE, internal bond, screw withdrawal, water absorption and thickness swelling for different treatments.

\begin{tabular}{c|c|c|c|c|c|c}
\hline Tratamentos & $\begin{array}{c}\text { MOR } \\
\left(\mathrm{kgf} / \mathrm{cm}^{2}\right)\end{array}$ & $\begin{array}{c}\text { MOE } \\
\left(\mathrm{kgf} / \mathrm{cm}^{2}\right)\end{array}$ & $\begin{array}{c}\mathrm{LI} \\
\left(\mathrm{kgf} / \mathrm{cm}^{2}\right)\end{array}$ & $\begin{array}{c}\text { AP } \\
(\mathrm{kgf})\end{array}$ & $\begin{array}{c}\text { Ab 24 } \\
(\%)\end{array}$ & $\begin{array}{c}\text { IE 24 } \\
(\%)\end{array}$ \\
\hline 1 & 38,08 & 5048 & 0,46 & 39 & 73,21 & 41,04 \\
2 & 52,79 & 7248 & 1,41 & 62 & 65,79 & 29,43 \\
3 & 59,83 & 7550 & 2,13 & 55 & 78,20 & 32,03 \\
4 & 51,89 & 7820 & 0,76 & 49 & 64,76 & 45,56 \\
5 & 86,29 & 10584 & 1,75 & 70 & 57,09 & 31,84 \\
6 & 83,65 & 12633 & 2,41 & 72 & 57,48 & 26,49 \\
7 & 74,41 & 12977 & 0,95 & 60 & 58,04 & 47,99 \\
8 & 95,84 & 16206 & 1,45 & 72 & 51,13 & 29,05 \\
9 & 95,71 & 15970 & 3,04 & 80 & 46,81 & 21,63 \\
10 & 42,76 & 7351 & 0,36 & 34 & 81,48 & 45,26 \\
11 & 55,08 & 10143 & 1,59 & 49 & 65,11 & 26,67 \\
12 & 54,55 & 7989 & 2,72 & 47 & 52,30 & 18,90 \\
13 & 53,99 & 8546 & 1,05 & 48 & 65,13 & 35,24 \\
14 & 63,61 & 10154 & 1,35 & 53 & 62,79 & 38,84 \\
15 & 94,17 & 12749 & 2,32 & 80 & 54,50 & 23,59 \\
16 & 68,63 & 12147 & 1,19 & 58 & 60,20 & 37,51 \\
17 & 100,86 & 14774 & 1,97 & 71 & 53,64 & 27,39 \\
18 & 100,44 & 18574 & 2,90 & 65 & 41,05 & 20,58 \\
\hline
\end{tabular}

Em que: $\mathrm{MOR}=$ módulo de ruptura $\left(\mathrm{kgf} / \mathrm{cm}^{2}\right) ; \mathrm{MOE}=$ módulo de elasticidade $\left(\mathrm{kgf} / \mathrm{cm}^{2}\right) ; \mathrm{LI}=$ ligação interna $\left(\mathrm{kgf} / \mathrm{cm}^{2}\right) ; \mathrm{AP}=$ resistência ao arrancamento de parafusos (kgf); Ab24 = absorção em peso 24 horas de imersão; IE24 = inchamento em espessura 24 horas de imersão.

A correlação entre as propriedades de flexão estática das chapas, definidas pelo MOR e o MOE, e as variáveis independentes analisadas (tipo de partícula, teor de adesivos, densidade observada) são apresentadas na Tabela 3. A correlação de Pearson evidencia que o MOR e o MOE apresentaram valores significativos para todas as variáveis independentes analisadas. Os valores de correlação explicam que, quando diminui a proporção de cavacos aumentando-se a proporção de adesivo e densidade, eleva-se o valor do MOR e do MOE.

TABELA 3: Correlação de Pearson entre as propriedades de flexão estática e as variáveis de fabricação das chapas para partícula tipo palito e serragem.

TABLE 3: Pearson correlations between the bending properties and the manufacturing parameters for particles type sticks and sawdust.

\begin{tabular}{c|c|c|c|c|c}
\hline TP & Correlações & $\begin{array}{c}\text { Teor de adesivo } \\
(\%)\end{array}$ & $\begin{array}{c}\text { Densidade observada } \\
\left(\mathrm{g} / \mathrm{cm}^{3}\right)\end{array}$ & $\begin{array}{c}\text { MOR } \\
\left(\mathrm{kgf} / \mathrm{cm}^{2}\right)\end{array}$ & $\begin{array}{c}\text { MOE } \\
\left(\mathrm{kgf} / \mathrm{cm}^{2}\right)\end{array}$ \\
\hline Palito & MOR $\left(\mathrm{kgf} / \mathrm{cm}^{2}\right)$ & $0,427(* *)$ & $0,598(* *)$ & 1 & \\
Palito & MOE $\left(\mathrm{kgf} / \mathrm{cm}^{2}\right)$ & $0,314(* *)$ & $0,530(* *)$ & $0,859(* *)$ & 1 \\
Serragem & MOR $\left(\mathrm{kgf} / \mathrm{cm}^{2}\right)$ & $0,464(* *)$ & $0,733(* *)$ & 1 & 1 \\
Serragem & MOE $\left(\mathrm{kgf} / \mathrm{cm}^{2}\right)$ & $0,386(* *)$ & $0,643(* *)$ & $0,822(* *)$ & 1 \\
\hline
\end{tabular}

Em que: TP $=$ tipo de partícula; ${ }^{* *}$ Correlação significativa a $1 \%$ de probabilidade; ns $=$ correlação não-significativa a $5 \%$ de probabilidade; $\mathrm{MOR}=$ módulo de ruptura; $\mathrm{MOE}=$ módulo de elasticidade.

A correlação de Pearson (Tabela 3) evidencia que o MOR e o MOE apresentaram valores significativos para todas as variáveis independentes analisadas. Os valores de correlação explicam que, quando diminui a proporção de cavacos aumentando-se a proporção de adesivo e densidade, eleva-se o valor do MOR e do MOE.

Observou-se também neste estudo que a variável que melhor se relacionou com o MOR e o MOE foi a densidade real das chapas produzidas com tipo de partícula serragem. Isso também foi verificado por Hillig 
(2000), que a densidade da chapa obteve valor de correlação elevado para MOR e MOE .

As Figuras 1 e 2 ilustram as relações de MOR e MOE, respectivamente ajustados em função da densidade e do teor de adesivo respectivamente.
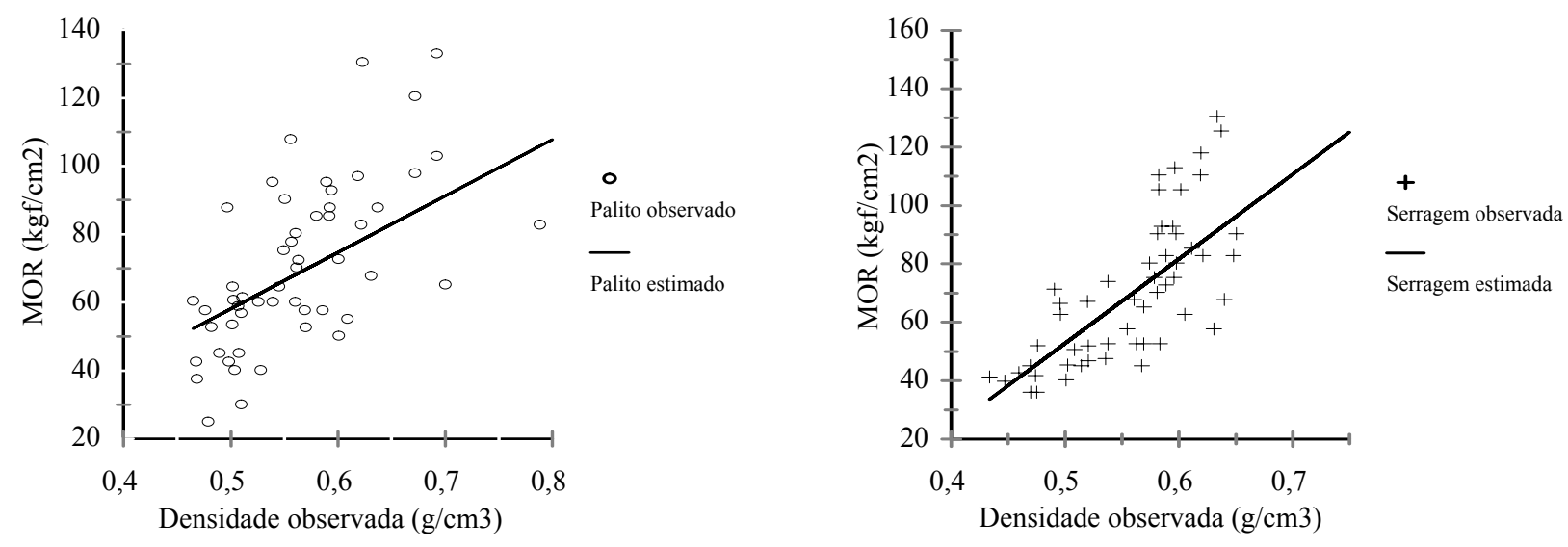

FIGURA 1: Valores de MOR observados e estimados em função da densidade observada, teor de adesivo igual a $8 \%$, partícula tipo palito e serragem respectivamente.

FIGURE 1: MOR observed and fitted as a function of board density, adhesive content of $12 \%$ and type of particles sticks and sawdust, respectively.
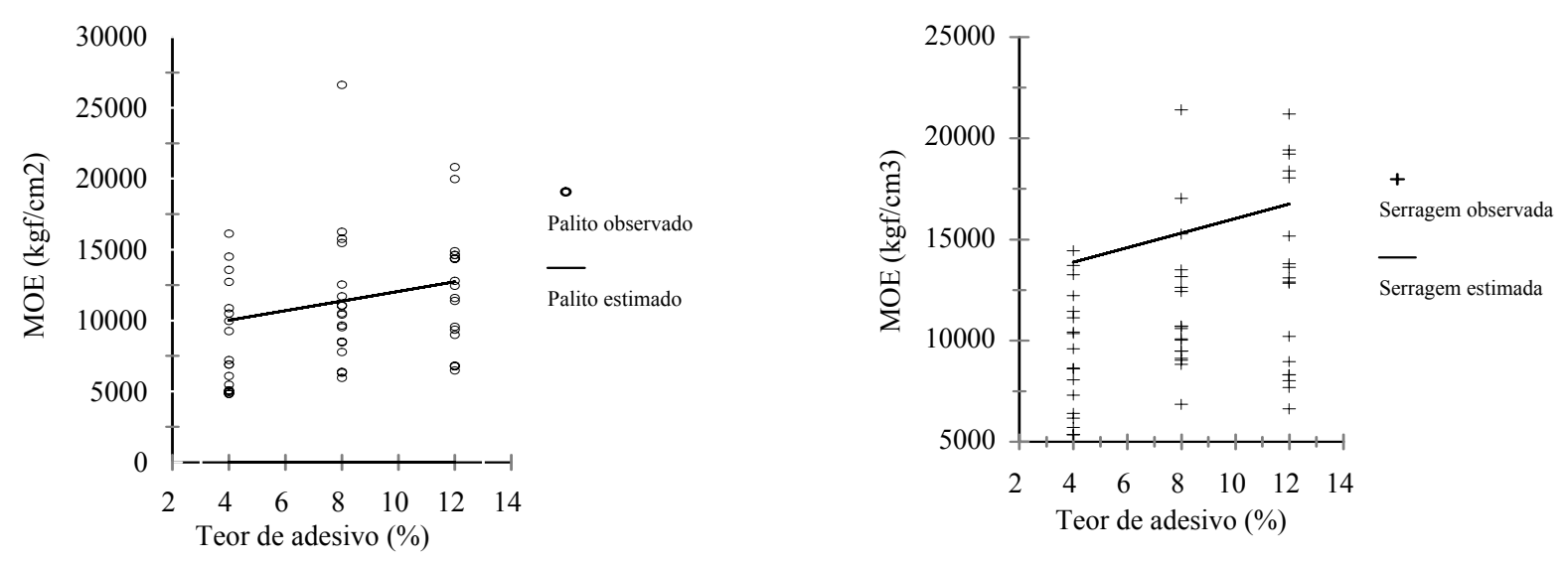

FIGURA 2: Valores de MOE observados e ajustados em função do teor de adesivo, densidade igual a 0,65 $\mathrm{g} / \mathrm{cm}^{3}$, partícula tipo palito e serragem respectivamente.

FIGURE 2: MOE observed and fitted as a function of adhesive content, board density of $0,65 \mathrm{~g} / \mathrm{cm}^{3}$, and type of particles sticks and sawdust, respectively.

As equações ajustadas de MOR, MOE, ligação interna, arrancamento de parafusos, absorção d'água e inchamento em espessura em função do teor de adesivo e densidade, tanto para chapas produzidas com partículas tipo palitos quanto serragem, podem ser vistas na Tabela 4. 
TABELA 4: Equações ajustadas para as propriedades das chapas em função das variáveis de fabricação para partícula tipo palito e serragem.

TABLE 4: Fitted equations for the properties of the board as a function of manufacturing variables for particles type sticks and sawdust.

\begin{tabular}{cll|c|c|c}
\hline TP & \multicolumn{1}{c|}{ Equação ajustada } & $\mathrm{F}$ & Syx & $\mathrm{R}^{2}$ aj. $(\%)$ & $\mathrm{DW}$ \\
\hline Palito & $\sqrt{M O R}=1,85883+0,13575 *^{*} \operatorname{tad}+9,42257 * \mathrm{Do}$ & 21,26 & 1,07 & 43,32 & 1,155 \\
Palito & $\sqrt{M O E}=13,093+1.56985 * \operatorname{tad}+124,111 * \mathrm{Do}$ & 16,26 & 0,16 & 36,54 & 0,86 \\
Palito & $\sqrt{L I}=-0,945833+2,74726 * \mathrm{Do}+0,0844171 * \operatorname{tad}$ & 157,4 & 0,23 & 70,00 & 1,28 \\
Palito & $\sqrt{A P}=3,62317+5,01909 * \mathrm{Do}+0,117331 * \operatorname{tad}$ & 12,75 & 0,89 & 30,72 & 1,15 \\
Palito & $\sqrt{A b 24}=13,2999-9,96154 * \mathrm{Do}$ & 35,37 & 0,60 & 40,48 & 1,62 \\
Palito & $\sqrt{I E 24}=7,3321-0,198147 * \operatorname{tad}$ & 71,57 & 0,56 & 57,91 & 1,29 \\
Serragem & $\sqrt{M O R}=91719+0,151872 * \operatorname{tad}+17,8852 * \mathrm{Do}$ & 52,32 & 0,83 & 66,80 & 1,65 \\
Serragem & $\sqrt{M O E}=-16,0532+1,44905 * \operatorname{tad}+197,148 * \mathrm{Do}$ & 25,04 & 12,97 & 48,52 & 1,12 \\
Serragem & $\sqrt{L I}=-0,286142+1,581 * \mathrm{Do}+0,085884 * \operatorname{tad}$ & 93,00 & 0,261 & 57,86 & 0,99 \\
Serragem & $\sqrt{A P}=2,19916+8,0614 * \mathrm{Do}+0,139095 * \operatorname{tad}$ & 19,98 & 0,81 & 41,73 & 1,75 \\
Serragem & $\sqrt{A b 24}=8,92045-0,159994 * \operatorname{tad}$ & 46,32 & 0,56 & 47,11 & 0,71 \\
Serragem & $\sqrt{I E 24}=7,09529-0,209764 * \operatorname{tad}$ & 133,9 & 0,43 & 72,03 & 1,38
\end{tabular}

Em que: TP = tipo de partícula; Tad = teor de adesivo(\%); Do = densidade observada $\left(\mathrm{g} / \mathrm{cm}^{3}\right) ; \mathrm{MOR}=$ módulo de ruptura $\left(\mathrm{kgf} / \mathrm{cm}^{2}\right) ; \mathrm{MOE}=$ módulo de elasticidade $\left(\mathrm{kgf} / \mathrm{cm}^{2}\right) ; \mathrm{LI}=$ ligação interna(kgf); $\mathrm{AP}=$ resistência ao arrancamento de parafuso (kgf); Ab 24 = absorção em peso 24 horas de imersão (\%); IE 24 = inchamento em espessura 24 horas de imersão; $F$ = teste de F; Syx = erro-padrão da estimativa; $\mathrm{R}^{2}$ aj = coeficientes de determinação; $\mathrm{DW}=$ teste de Durbin-Watson.

Nos ensaios de ligação interna, foi observado que os maiores valores foram conseguidos com as chapas produzidas com $12 \%$ de adesivo, e os mais baixos, com aquelas produzidas com teor de adesivo de $4 \%$. Dessa maneira, pode-se concluir que um dos fatores responsáveis pela baixa resistência de colagem é o teor de adesivo, tanto para palitos quanto para serragem.

Dacosta (2004) obteve o maior valor de ligação interna com o uso do adesivo em proporção de $12 \%$, independentemente do tipo de resíduo.

Além do teor de adesivo, outro fator que também influenciou nos valores de ligação interna foi a densidade das chapas. As chapas produzidas com densidade $0,75 \mathrm{~g} / \mathrm{cm}^{3}$ apresentaram os melhores valores. Já para as produzidas com $0,55 \mathrm{~g} / \mathrm{cm}^{3}$, foram encontrados valores bem menores de ligação interna.

Da mesma forma, Alberto \& Keinert Jr. (1993) observaram também que chapas mais densas apresentam valores mais altos de ligação interna.

As correlações entre a propriedade de ligação interna, o teor de adesivo e a densidade da chapa para partícula tipo palito e serragem podem ser vistas na Tabela 5 .

TABELA 5: Correlação de Pearson entre a propriedade de ligação interna e as variáveis de fabricação das chapas para partícula tipo palito e serragem.

TABLE 5: Pearson correlations between the internal bond and the manufacturing parameters for particles type sticks and sawdust.

\begin{tabular}{c|c|c|c|c}
\hline TP & Correlações & Teor de adesivo (\%) & Densidade observada $\left(\mathrm{g} / \mathrm{cm}^{3}\right)$ & LI $\left(\mathrm{kgf} / \mathrm{cm}^{2}\right)$ \\
\hline Palito & LI $\left(\mathrm{kgf} / \mathrm{cm}^{2}\right)$ & $0,732(* *)$ & $0,530(* *)$ & 1 \\
Serragem & LI $\left(\mathrm{kgf} / \mathrm{cm}^{2}\right)$ & $0,728(* *)$ & $0,307(* *)$ & 1 \\
\hline
\end{tabular}

Em que: TP = tipo de partícula; $* *=$ Correlação significativa a $1 \%$ de probabilidade; ns = correlação não-significativa a $5 \%$ de probabilidade; $\mathrm{LI}=$ ligação interna.

A Tabela 5 evidencia que a ligação interna apresentou valores significativos de correlação para as variáveis, indicando que, com o aumento do teor de adesivo e da densidade, a ligação interna aumenta na 
chapa.

Os melhores modelos de regressão para LI para chapas produzidas com partícula tipo palito e serragem são apresentado na Tabela 4.

As Figuras 3 e 4 mostram os gráficos com os valores ajustados para a densidade observada e o teor de adesivo respectivamente.
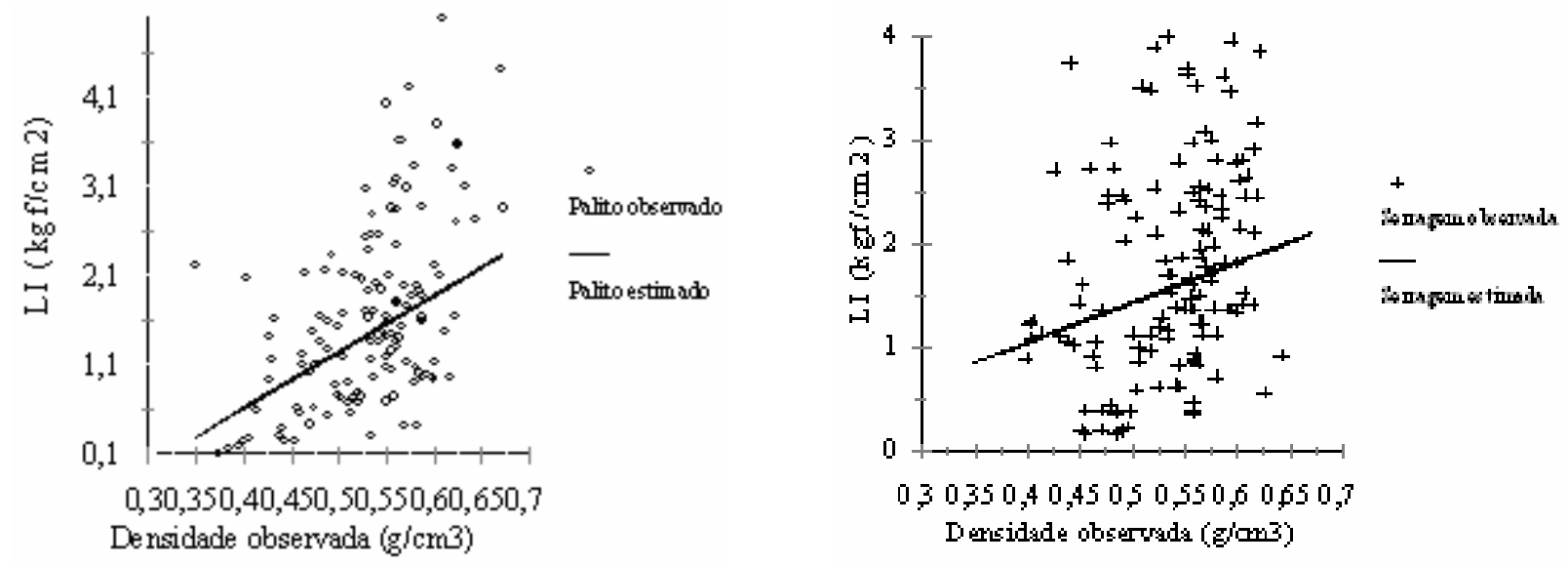

FIGURA 3: Valores de ligação interna, observados e estimados em relação à densidade, teor de adesivo de $8 \%$ e partícula tipo palito e serragem respectivamente.

FIGURE 3: Internal bond observed and fitted as a function of board density, adhesive content of $8 \%$, and type of particles sticks and sawdust, respectively.
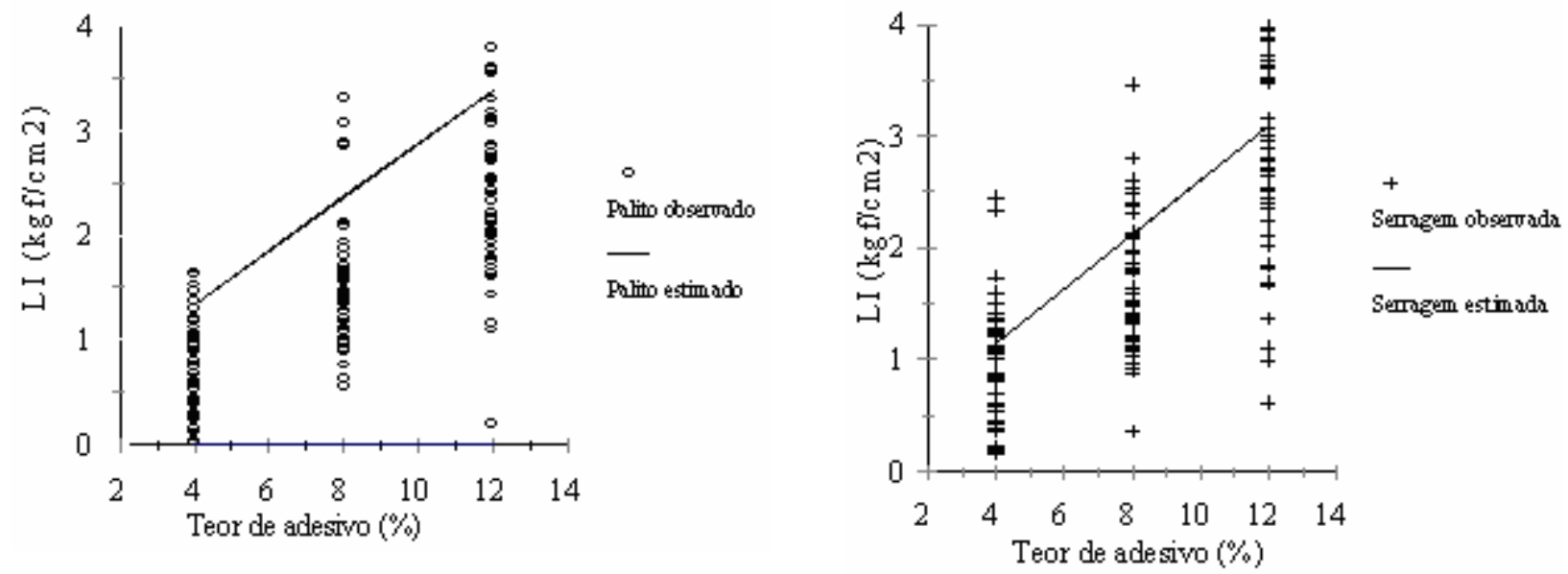

FIGURA 4: Valores de ligação interna, observados e estimados em função do teor de adesivo, densidade de $0,65 \mathrm{~g} / \mathrm{cm}^{3}$ partícula tipo palito e serragem respectivamente.

FIGURE 4: Internal bond observed and fitted as a function of adhesive content, board density of $0.65 \mathrm{~g} / \mathrm{cm}^{3}$, and type of particles sticks and sawdust, respectively.

O teor de adesivo e a densidade das chapas também influenciaram nas resistência ao arrancamento de parafusos, pois, com o aumento da quantidade de adesivo aplicado nas chapas, os valores de resistência também aumentaram. parafuso.

O tipo de partícula (palito e serragem) utilizada não influenciou na resistência ao arrancamento de

Resultados semelhantes foram obtidos por Vital et al. (1992) em que a resistência ao arrancamento de parafuso em chapas de partículas e de flocos não foi afetada pela geometria da matéria-prima.

As correlações entre a propriedade de resistência ao arrancamento de parafuso da chapa e as variáveis analisadas para partícula tipo palito e serragem podem ser vistas na Tabela 6 . 
TABELA 6: Correlação de Pearson entre a propriedade de resistência ao arrancamento de parafusos e as variáveis de fabricação das chapas para partícula tipo palito e serragem.

TABLE 6: Pearson correlations between screw withdrawal resistance and the manufacturing parameters for particles type sticks and sawdust.

\begin{tabular}{cccccc}
\hline TP & Correlações & Teor de adesivo (\%) & Densidade observada $\left(\mathrm{g} / \mathrm{cm}^{3}\right)$ & AP $(\mathrm{kgf})$ \\
\hline Palito & AP (kgf) & $0,428(* *)$ & $0,603(* *)$ & 1 \\
Serragem & AP (kgf) & $0,457(* *)$ & $0,758(* *)$ & 1 \\
\hline
\end{tabular}

Em que: TP $=$ tipo de partícula; ${ }^{* *}$ Correlação significativa a $1 \%$ de probabilidade; $n s=$ correlação não-significativa a $5 \%$ de probabilidade; $\mathrm{AP}=$ arrancamento de parafusos.

Como observado na Tabela 6, as correlações comprovam que, aumentando o teor de adesivo e a densidade, ocorre um aumento na resistência no arrancamento de parafusos. Os modelos de regressão linear utilizados para os dois tipos de partículas (palitos e serragem) incluem o teor de adesivo e a densidade.

A Figura 5 mostra os gráficos com os valores de teor de adesivo e resistência ao arrancamento de parafusos ajustados para partícula tipo palito e serragem.
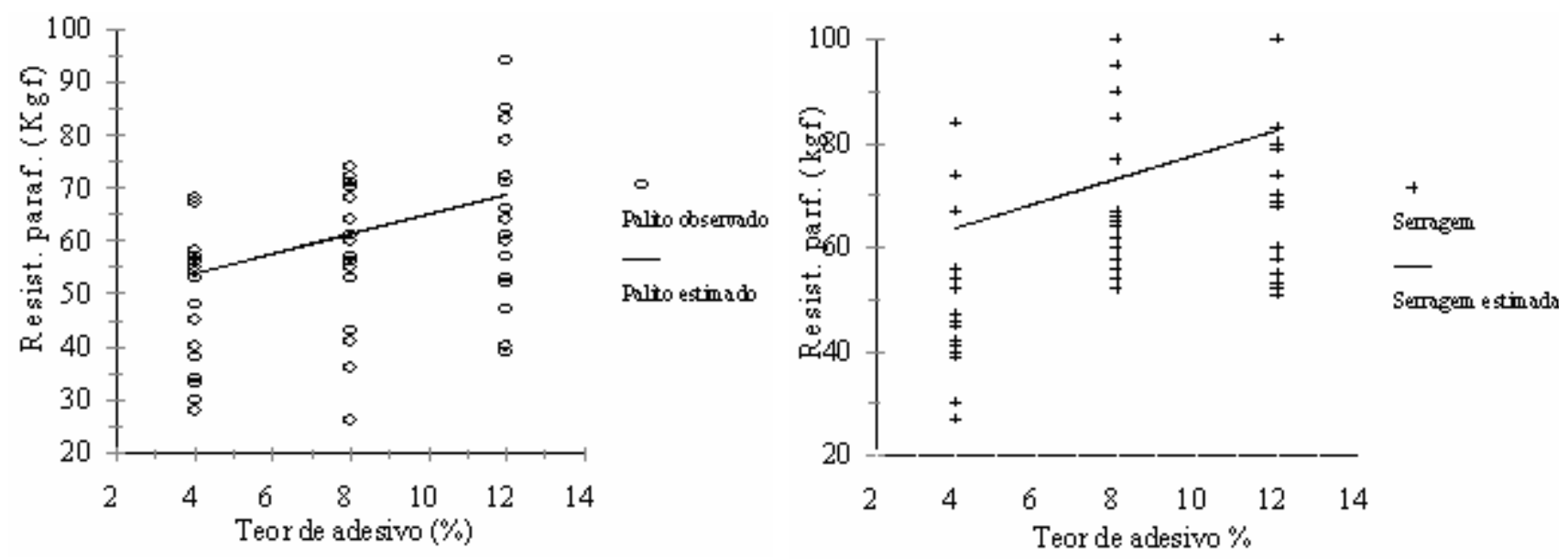

FIGURA 5: Valores de resistência ao arrancamento de parafuso observados e estimados em função do teor de adesivo, densidade de $0,65 \mathrm{~g} / \mathrm{cm}^{3}$ e partícula tipo palito e serragem respectivamente.

FIGURE 5: Screw withdrawal properties observed and adjusted according to adhesive content, board density of $0.65 \mathrm{~g} / \mathrm{cm}^{3}$, and type of particles sticks and sawdust, respectively.

\section{Propriedades físicas}

\section{Absorção d'água e inchamento em epessura}

Para os ensaios de absorção d'água e inchamento em espessura, os menores valores foram encontrados para os tratamentos que utilizaram $12 \%$ de adesivo. Já os tratamentos com apenas $4 \%$ de adesivo obtiveram os maiores valores de absorção d'água e inchamento em espessura. Observou-se também que, com a redução da densidade, os valores de absorção aumentaram. Isso também foi verificado por Dacosta (2004) que constatou que as chapas com menor quantidade de adesivo e de menores densidades foram as que obtiveram os maiores valores de absorção d'água.

As correlações entre as propriedades de estabilidade dimensional e as variáveis de fabricação das chapas para partícula tipo palito e serragem podem ser vistas na Tabela 7.

Como observado na Tabela 7, aumentando o teor de adesivo e da densidade, diminuiu os valores de absorção d'água e o inchamento em espessura das chapas. Para chapas produzidas com partícula tipo serragem, apenas o teor de adesivo obteve correlação significativa. 
TABELA 7: Correlação de Pearson entre as propriedades de estabilidade dimensional e as variáveis de fabricação das chapas para partícula tipo palito e serragem.

TABLE 7: Pearson correlations between the dimensional stability properties and the manufacturing parameters for particles type sticks and sawdust.

\begin{tabular}{ccccccc}
\hline TP & Correlações & Teor de adesivo (\%) & Densidade observada $\left(\mathrm{g} / \mathrm{cm}^{3}\right)$ & Ab 24 (\%) & IE 24 (\%) \\
\hline Palito & Ab 24 (\%) & $-0,148(\mathrm{~ns})$ & $-0,614(* *)$ & 1 & \\
Palito & IE 24 (\%) & $0,746\left(^{*}\right)$ & $-0,223(\mathrm{~ns})$ & $0,419(* *)$ & 1 \\
Serragem & Ab 24 (\%) & $-0,664(* *)$ & $-0,277(*)$ & 1 & $0,718(* *)$ \\
Serragem & IE 24 (\%) & $-0,837(* *)$ & $0,028(\mathrm{~ns})$ & & 1 \\
\hline
\end{tabular}

Em que: TP = tipo de partícula; ${ }^{* *}$ Correlação significativa a $1 \%$ de probabilidade; $n s=$ correlação não-significativa a $5 \%$ de probabilidade; Ab24 = absorção em peso 24 horas de imersão; IE24 = inchamento em espessura 24 horas de imersão.

A Figura 6 mostra os gráficos para absorção de água e inchamento em espessura com os valores ajustados em função do teor de adesivo.

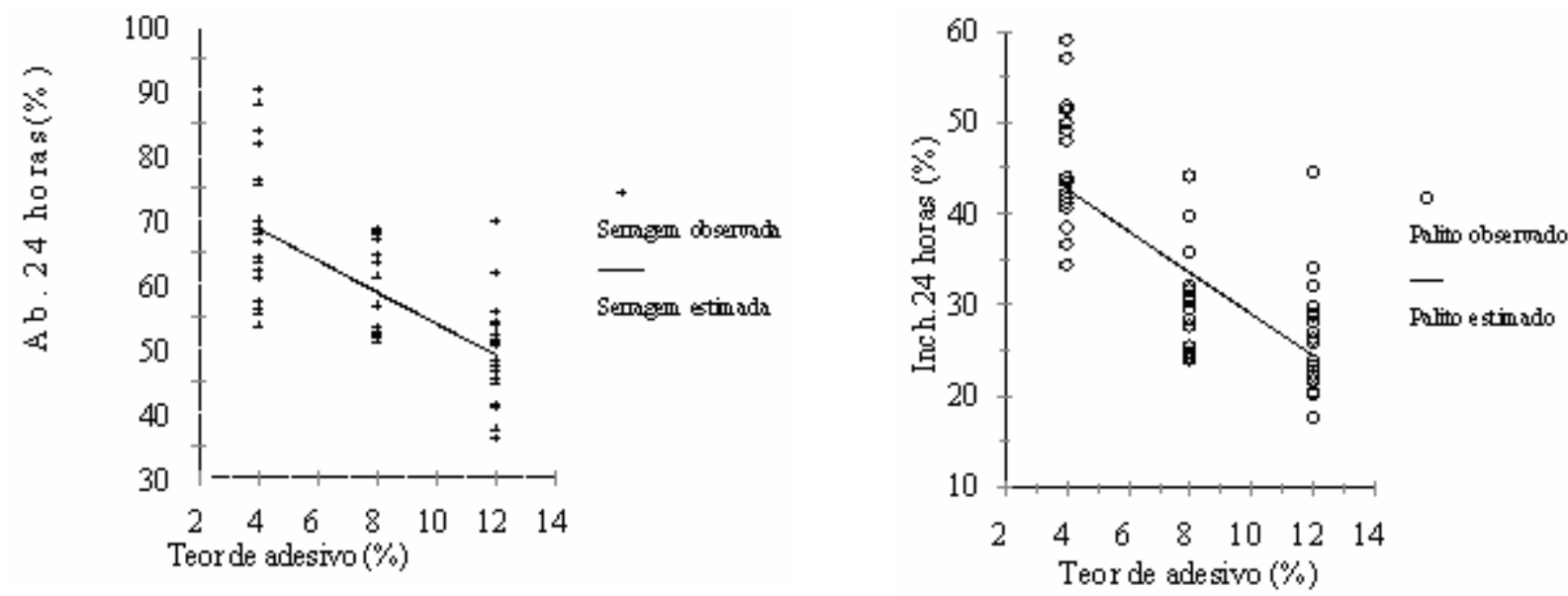

FIGURA 6: Valores de absorção d'água e inchamento em espessura observados e estimados em função do teor de adesivo para partícula tipo serragem e palito, respectivamente.

FIGURE 6: Water absorption and thickness swelling observed and adjusted according to adhesive content, for type of particles sawdust and sticks, respectively.

\section{CONCLUSÕES}

Os resultados obtidos neste estudo permitem as seguintes conclusões:

De um modo geral, chapas aglomeradas produzidas tanto com partículas tipo palito como serragem apresentaram boa qualidade, evidenciando a viabilidade de uso desses resíduos na fabricação desse produto, e com isso possibilitando novas alternativas de aproveitamento desse material pela indústria.

Os valores médios encontrados para módulo de ruptura (MOR) e módulo de elasticidade (MOE), na maioria dos tratamentos, ficaram dentro dos exigidos pelos padrões de qualidade internacional, tanto para partículas tipo palito como para serragem.

Dentre as variáveis estudadas, a densidade da chapa foi a que mais influenciou no módulo de ruptura e no módulo de elasticidade, seguida do teor de adesivo e do tipo de partícula.

Os valores médios para ligação interna ficaram dentro dos limites determinados pelos padrões de qualidade internacional. $\mathrm{O}$ teor de adesivo foi o parâmetro que mais influenciou essa propriedade, seguido da densidade da chapa.

$\mathrm{Na}$ resistência ao arrancamento de parafuso, os valores obtidos atingiram os valores exigidos pelos padrões de qualidade internacional, em alguns tratamentos. Para essa propriedade, a densidade das chapas foi a variável que teve maior influência, seguida do teor de adesivo.

Para a absorção d'água e inchamento em espessura, foi observado que a variável que exerceu maior 
influência foi o teor de adesivo: a absorção d'água e o inchamento em espessura diminuíram com o aumento do teor de adesivo.

De forma geral, não foram observadas mudanças na qualidade das chapas com relação ao tipo de resíduo utilizado, sendo encontrados valores muito semelhantes na maioria das propriedades analisadas para chapas fabricadas com partícula tipo palito e serragem.

Com base nos parâmetros de fabricação as chapas com densidade de $0,75 \mathrm{~g} / \mathrm{cm}^{3}$ produzidas com $12 \%$ de adesivo, tanto de palito como de serragem, estão mais adequadas às normas de comercialização.

\section{AGRADECIMENTOS}

Os autores agradecem às empresas Klabin Riocell de Guaíba-RS, Synteko de Gravataí-RS, pelo fornecimento das partículas de madeira e do adesivo respectivamente.

\section{REFERÊNCIAS BIBLIOGRÁFICAS}

ALBERTO, M. M. M.; KEINERT JR., S. Efeito da geometria das partículas e da densidade, sobre as propriedades de painéis estruturais "waferboards" : parte I : efeito sobre as propriedades de resistência. In: CONGRESSO FLORESTAL PANAMERICANO, 1., 1993, Curitiba. Anais... Curitiba, 1993. p. 613.

AMERICAN NATIONAL STANDARDS INSTITUTE. Particleboard, ANSI A 208.1. New York, 1978.

AMERICAN SOCIETY FOR TESTING AND MATERIALS. Standard test methods for evaluating properties of wood-base fiber and particle panel materials. ASTM D 1037 - 93. Philadelphia, 1995.

AMERICAN SOCIETY FOR TESTING AND MATERIALS. Standard specification for urea-formaldehyde resin adhesives, D 4690-95a. Philadelphia, 1999.

BRITO, E. O. et al. Nos caminhos do eucaliptos. Bento Gonçalves : CETEMO, 1998. 88p. (Coletânea. SENAI-RS)

CALEGARI, L.; HASELEIN, C. R.; BARROS, M. V.; SCARAVELli, T. L.; DACOSTA, L. P.; PEDRAZZI, C. Adição de aparas de papel reciclável na fabricação de chapas de madeira aglomerada. Ciência Florestal, Santa Maria, v. 14, n. 1, p. 193-204, 2004.

DACOSTA, L.P.E. Utilização de resíduos do processamento mecânico da madeira para fabricação de chapas de partículas aglomeradas. 2004. 118p. Dissertação (Mestrado em Engenharia Florestal) - Universidade Federal de Santa Maria, Santa Maria, 2004.

FOELKEL, C.E.B.; COUTO, L.C.; KATO, J. A utilização da serragem de madeira de eucalipto na produção de polpa celulósica. Cenibra : São Paulo, 1979. 21p.

HASELEIN, C. R. Análise de parâmetros para a fabricação de chapas de partículas aglomeradas de madeira de eucalipto (Eucalyptus grandis W. Hill ex- Maiden) e embaúba (Cecropia sp.). Viçosa, 1989. 75f. Dissertação (Mestrado em Ciência Florestal) - Universidade Federal de Viçosa, Viçosa, 1989.

HILLIG, E. Qualidade de chapas aglomeradas estruturais, fabricadas com madeiras de Pinus, Eucalipto e Acácia negra, puras ou misturadas, coladas com tanino-formaldeído. 2000. 96f. Dissertação (Mestrado em Engenharia Florestal) - Universidade Federal de Santa Maria, Santa Maria, 2000.

IWAKIRI S. et al. Mistura de espécies na produção de chapas de partículas estruturais Waferboard. Agrárias, Curitiba, v.14, p.107-114, 1995.

IWAKIRI S.; PEREIRA, S.J.; NISGOSKI, S. Influência da umidade, pressão e tempo de prensagem na produção de chapas de partículas de madeira aglomerada . Revista Ciências Agrárias, Curitiba, v. 18, n. 1-2. p. 29-36.,1999a.

IWAKIRI S. et al. Utilização de resíduos de serraria na produção de chapas de madeira aglomerada de Eucalyptus saligna, Eucalyptus citriodora e Eucalyptus pilularis. Floresta e Ambiente, Rio de Janeiro, v. 7, n. 1, p. 251-256, 2000.

KELLY, M.W. Critical literature review of relationship between processing parameters and physical properties of particleboard. Madison : USDA, Forest Service, Forest Products Laboratory, 1977. 66f. (USDA Forest Service. Forest Products Laboratory General Technical Report FPL, v. 10)

KOCH, G.S.; KLAREICH, F.; EXTRUM, B. Adhesives for the composite wood panel industry. New Jersey : Noyes Data Corporation, 1987.

MALONEY, T.M. Moderm particleboard and dry-process fiberboard manufacturing. San Francisco: Miller Freeman, 1993. 681p.

MOSLEMI, A.A. Particleboard. Illinois : Southern. Illinois University Press, 1974. v. 2, 245p. 
PIZZI, A. Advanced wood adhesives technology. New York : Marcel Dekker, 1994. 370p.

TSOUMIS, G. Science and technology of wood : structure, properties, utilization. New York : Chapman \& Hall, 1991. 494p.

VITAL, B.R.; HASELEIN, C.R.; DELLALUCIA R.M. Efeito da geometria das partículas nas propriedades das chapas de madeira aglomerada de Eucalyptus grandis (Hill ex-Maiden). Revista Árvore,Viçosa, v. 16, n. 1, p. 88-96, 1992. 